\title{
Synthesis of a New $N$-Acetyl Thiazolidinethione Reagent and its Application to a Highly Selective Asymmetric Acetate Aldol Reaction
}

\author{
Yingchao Zhang and Tarek Sammakia* \\ Department of Chemistry, University of Colorado, Boulder, Colorado 80309-0215
}

Supporting Information

General Information. All non-aqueous reactions were conducted in oven-dried glassware, under a dry nitrogen atmosphere. Dichlorophenylborane (97\%) was purchased from Aldrich and distilled, then stored in a resealable container bearing a teflon valve at $-26{ }^{\circ} \mathrm{C}$ under a nitrogen atmosphere. $\mathrm{CeCl}_{3}(99+\%$, anhydrous, beads -10 mesh) was purchased from Aldrich and further dried under high vacuum at $140{ }^{\circ} \mathrm{C}$ (sand bath temperature) for $3 \mathrm{~h}$. (-)-Sparteine was distilled and stored under a nitrogen atmosphere at $-26^{\circ} \mathrm{C}$. All aldehydes were distilled from $\mathrm{MgSO}_{4}$ and $\mathrm{K}_{2} \mathrm{CO}_{3}$ and then used immediately. Acetyl chloride was freshly distilled under nitrogen immediately prior to use. $\mathrm{CH}_{2} \mathrm{Cl}_{2}$ was distilled from $\mathrm{CaH}_{2}$ under nitrogen. THF was distilled from $\mathrm{Na}$ benzophenone ketyl under nitrogen. $\mathrm{NEt}_{3}$ was distilled from $\mathrm{CaH}_{2}$ under nitrogen. All other starting materials were used as received. Flash chromatography of the aldol adducts was performed on neutral silica gel (Mallinckrodt Silicar Silica Gel 150, 60-200 mesh (75-250 micron)) following the procedure of Still. ${ }^{1}$ All other flash chromatography was performed using Sorbent Technologies 60 A silica gel (32-63 micron). Flash chromatography of the aldol adducts on this silica or other "ordinary" silica gel (i.e., silica gel with a $\mathrm{pH}$ other than 7.0) led to partial cleavage of the thiazolidinethinoe auxiliary. ${ }^{1} \mathrm{H}$ NMR spectra were recorded at $500 \mathrm{MHz}$ or $400 \mathrm{MHz}$, using $\mathrm{CDCl}_{3}$ (internal reference $7.24 \mathrm{ppm}$ ) as solvent. ${ }^{13} \mathrm{C}$ NMR spectra were obtained at $100 \mathrm{MHz}$ using $\mathrm{CDCl}_{3}$ (internal reference $77.23 \mathrm{ppm}$ ) as solvent. Chiral HPLC analysis was performed using a Chiralcel OD column equipped with a UV detector $(245 \mathrm{~nm})$. Infrared spectra were recorded as thin films on $\mathrm{NaCl}$ plates. Melting points were determined in capillaries and are uncorrected. Optical rotations were determined on a JASCO 1030 polarimeter at $27^{\circ} \mathrm{C}$. Combustion analyses were performed by NuMega Resonance Labs, Inc. of San Diego, CA. Exact mass was calculated on $\mathrm{M}+\mathrm{Na}$ using electrospray ionization.

\section{Thiazolidinethione 3}
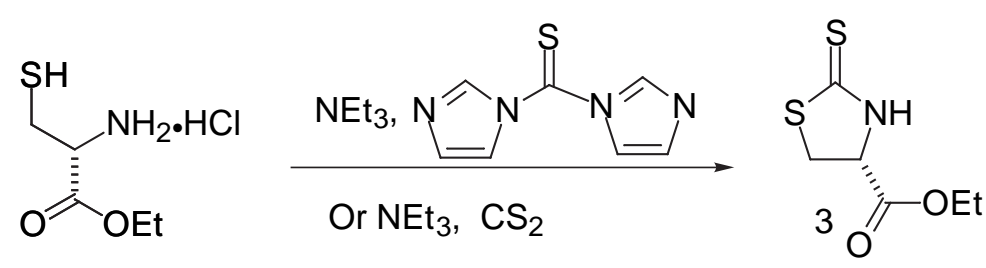

Thiazolidinethione $\mathbf{3}$ was synthesized by two different literature procedures as described

1 Still, W. C.; Kahn, M.; Mitra, A. J. Org. Chem. 1978, 43, 2923. 
below. $^{2}$

Procedure 1: Caution! This procedure uses the toxic reagent, $C S_{2}$. All manipulations must be conducted in a well ventilated fume hood, and care must taken to avoid contact or inhalation. To a suspension of L-cysteine ethyl ester hydrochloride $(5.0 \mathrm{~g}, 26.93 \mathrm{mmol})$ in methylene chloride $(40 \mathrm{~mL})$ under nitrogen at room temperature was added triethylamine $(3.75 \mathrm{~mL}$, $26.93 \mathrm{mmol})$ followed by carbon disulfide $(1.78 \mathrm{~mL}, 29.62 \mathrm{mmol})$. The reaction immediately turned a deep yellow color which faded over $10 \mathrm{~min}$ to a much lighter yellow suspension. The reaction mixture was stirred at room temperature overnight, then concentrated to a $10 \mathrm{~mL}$ suspension under reduced pressure. This suspension was directly subjected to purification by flash chromatography ( $\sim 80 \mathrm{~g}$ silica gel, $8: 1$ hexanes - ethylacetate) to provide thiazolidinethione $\mathbf{3}$ (3.86 $\mathrm{g}, 75 \%)$ as a light yellow oil.

Procedure 2: To a suspension of L-cysteine ethyl ester hydrochloride (5.0 g, $26.93 \mathrm{mmol})$ in THF $(40 \mathrm{~mL})$ under nitrogen at room temperature was added triethylamine $(3.75 \mathrm{~mL}, 26.93$ $\mathrm{mmol})$. The reaction was colled to $0{ }^{\circ} \mathrm{C}$, and the septum of the flask was removed briefly and $1,1^{\prime}-$ thiocarbonyldiimidazole $(4.80 \mathrm{~g}, 26.93 \mathrm{mmol})$ was added in one portion. The cold bath was removed, and the reaction was stirred for $1 \mathrm{~h}$. The reaction mixture was concentrated to a $10 \mathrm{~mL}$ suspension under reduced pressure. This suspension was directly subjected to purification by flash chromatography ( $\sim 80 \mathrm{~g}$ silica gel, 8:1 hexanes - ethylacetate) to provide thiazolidinethione $\mathbf{3}$ (4.63 $\mathrm{g}, 90 \%)$ as a light yellow oil.

${ }^{1} \mathrm{H}$ NMR $\left(500 \mathrm{MHz}, \mathrm{CDCl}_{3}\right): \delta 7.43$ (br s, $\left.1 \mathrm{H}\right), 4.78$ (X of ABX, 1H, J=7.30, $8.24 \mathrm{~Hz}$ ), $4.28(\mathrm{~m}, 2 \mathrm{H}), 3.79(\mathrm{~A}$ of $\mathrm{ABX}, 1 \mathrm{H}, \mathrm{J}=7.30,11.50 \mathrm{~Hz}), 3.78(\mathrm{~B}$ of $\mathrm{ABX}, 1 \mathrm{H}, \mathrm{J}=8.24,11.50 \mathrm{~Hz}), 1.31$ (t, 3H, J=7.15 Hz). ${ }^{13} \mathrm{C}$ NMR $\left(400 \mathrm{MHz}, \mathrm{CDCl}_{3}\right): \delta 201.5,168.5,64.0,63.0,35.7,14.3$. IR $\left(\mathrm{cm}^{-}\right.$ $\left.{ }^{1}\right): 3336,1654,1056 .[\alpha]_{D}=-49.5(\mathrm{c} 0.46$, EtOH $)$. HRMS m/z calc'd for $\mathrm{C}_{6} \mathrm{H}_{9} \mathrm{NO}_{2} \mathrm{~S}_{2} \mathrm{Na}^{+}$: 213.9967; found: 213.9966.

\section{Thiazolidinethione 4}

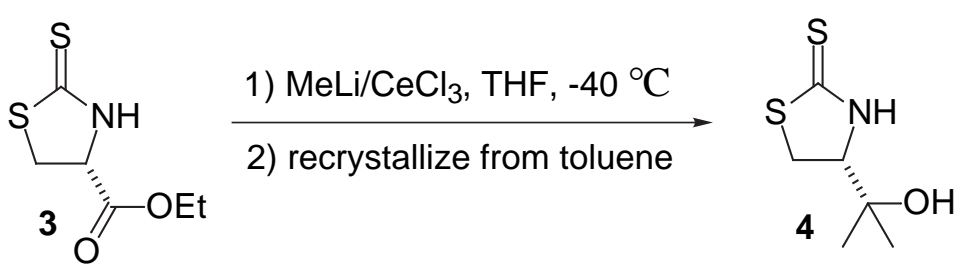

Anhydrous cerium chloride (30.93 g, $125.49 \mathrm{mmol}$ ) was further dried at $140{ }^{\circ} \mathrm{C}$ (sand bath temperature) for $3 \mathrm{~h}$ at high vacuum. Anhydrous THF $(150 \mathrm{~mL})$ was added by cannula, and the mixture was stirred at $\mathrm{rt}$ for $2 \mathrm{~h}$. The reaction mixture was cooled to $-78{ }^{\circ} \mathrm{C}$ and $\mathrm{MeLi}(84.5 \mathrm{~mL}$, $109.8 \mathrm{mmol}, 1.3 \mathrm{M}$ in $\mathrm{Et}_{2} \mathrm{O}$ ) was added dropwise by cannula. The resulting yellow creamy mixture was stirred at $-78{ }^{\circ} \mathrm{C}$ for $1 \mathrm{~h}$ and then warmed to $-40{ }^{\circ} \mathrm{C}$. To this suspension was added thiazolidinethione 3 ( $6 \mathrm{~g}, 31.37 \mathrm{mmol}$, solution in $20 \mathrm{~mL}$ THF) dropwise by cannula over a period of 20 min. The flask containing thiazolidinethione 3 was rinsed with 2 × $5 \mathrm{~mL}$ THF, which was also added to the reaction. The reaction was stirred at $-40{ }^{\circ} \mathrm{C}$ for additional 10 min and then quenched by the addition of $1 \mathrm{M} \mathrm{HCl}$ until all the solids dissolved (about $20 \mathrm{~mL}$ ). The layers were separated, and the aqueous layer was further extracted with $\mathrm{CHCl}_{3}(2 \times 200 \mathrm{~mL})$ and EtOAc $(100$ $\mathrm{mL}$ ). The combined organic layers were dried over anhydrous $\mathrm{MgSO}_{4}$, filtered, and then concentrated at reduced pressure to a white solid. Purification by recrystallization from toluene gave thiazolidinethione $\mathbf{4}$ as a white crystalline solid $(4.45 \mathrm{~g}, 80 \%, 99.4 \%$ ee). The enantiomeric

2 These procedure use readily available and cheap starting materials, $\mathrm{CS}_{2}$ and triethylamine, and afford the desired compound in good yield. However, they suffer from the toxicity and foul odor of $\mathrm{CS}_{2}$ : (a) Rufino, A. R.; Biaggio, F. C. Tetrahedron Lett. 2001, 42, 8559; (b) Nagao, Y.; Ikeda, T.; Inoue, T.; Yagi, M.; Shiro, M.; Fujita, E. J. Org. Chem 1985, 50, 4072; (c) Hsiao, C. N.; Liu, L.; Miller, M. J. Org. Chem. 1987, 52, 2201. This procedure uses a more expensive starting material, (1,1'-thiocarbonyldiimidazole), rather than $\mathrm{CS}_{2}$, and affords the desired compound in excellent yield: (d) Soai, K.; Ishizaki, M. Heterocycles 1984, 22, 2827. 
excess of this material was determined by conversion to thiazolidinethione $\mathbf{2}$, and analysis of this material by chiral HPLC (see experimental procedure for the synthesis of $\mathbf{2}$ for details).

${ }^{1} \mathrm{H} \mathrm{NMR}\left(500 \mathrm{MHz}, \mathrm{CDCl}_{3}\right): \delta 8.12$ (br s, $\left.1 \mathrm{H}\right), 4.22$ (ddd, $1 \mathrm{H}, \mathrm{J}=0.83,8.80,9.43 \mathrm{~Hz}$ ), 3.48( $\mathrm{A}$ of $\mathrm{ABX}, 1 \mathrm{H}, \mathrm{J}=9.43,11.35 \mathrm{~Hz}), 3.32(\mathrm{~B}$ of $\mathrm{ABX}, 1 \mathrm{H}, \mathrm{J}=8.80,11.35 \mathrm{~Hz}), 2.86(\mathrm{~s}, 1 \mathrm{H}), 1.30$ $(\mathrm{s}, 6 \mathrm{H}) .{ }^{13} \mathrm{C} \mathrm{NMR}\left(400 \mathrm{MHz}, \mathrm{CDCl}_{3}\right): \delta 201.7,73.0,72.8,34.2,27.5,23.9$. IR $\left(\mathrm{cm}^{-1}\right): 3440$, 3405, 1044. m.p. $=134 \sim 135{ }^{\circ} \mathrm{C}$. $[\alpha]_{\mathrm{D}}=+33.61$ (c 1.16, EtOH). HRMS m/z calc'd for $\mathrm{C}_{6} \mathrm{H}_{11} \mathrm{NOS}_{2} \mathrm{Na}^{+}:$200.0174; found: 200.0165 .

\section{Thiazolidinethione 5}
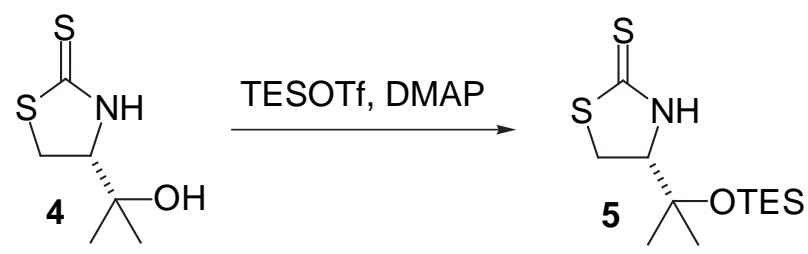

Thiazolidinethione 4 (4.45 g, $25.09 \mathrm{mmol})$ was suspended in $\mathrm{CH}_{2} \mathrm{Cl}_{2}(50 \mathrm{~mL})$ and cooled to $0{ }^{\circ} \mathrm{C}$. TESOTf $(11.35 \mathrm{~mL}, 50.20 \mathrm{mmol})$ was then added dropwise. After $20 \mathrm{~min}$, the heterogeneous reaction mixture turned to a yellow clear solution, at which point, the septum of the flask was removed briefly and DMAP $(6.13 \mathrm{~g}, 50.20 \mathrm{mmol})$ was added in one portion. The reaction was stirred at $0{ }^{\circ} \mathrm{C}$ for $20 \mathrm{~min}$, and then the ice bath was removed and the reaction was stirred for $40 \mathrm{~min}$. The reaction was then concentrated to a volume of $10 \mathrm{~mL}$ which was directly subjected to purification by flash chromatography (10:1 hexanes - ethylacetate) to provide thiazolidinethione $\mathbf{5}(6.97 \mathrm{~g}, 95 \%)$ as a colorless oil.

${ }^{1} \mathrm{H} \mathrm{NMR}\left(500 \mathrm{MHz}, \mathrm{CDCl}_{3}\right): \delta 7.35$ (br s, $\left.1 \mathrm{H}\right), 4.12(\mathrm{ddd}, 1 \mathrm{H}, \mathrm{J}=1.09,8.30,9.00 \mathrm{~Hz})$, 3.44(A of ABX, 1H, J=9.00, 11.35 Hz), 3.33 (B of ABX, 1H, J=8.30, 11.35 Hz), $1.25(\mathrm{~s}, 6 \mathrm{H}), 0.93$ $(\mathrm{t}, 9 \mathrm{H}, \mathrm{J}=8.00 \mathrm{~Hz}), 0.59(\mathrm{q}, 6 \mathrm{H}, \mathrm{J}=8.00 \mathrm{~Hz}) .{ }^{13} \mathrm{C} \mathrm{NMR}\left(400 \mathrm{MHz}, \mathrm{CDCl}_{3}\right): \delta 201.3,75.1,73.8$, 34.3, 26.4, 24.8, 7.24, 6.75. IR $\left(\mathrm{cm}^{-1}\right): 3351,1049 .[\alpha]_{\mathrm{D}}=-11.17(\mathrm{c} 0.42, \mathrm{EtOH}) . \mathrm{HRMS} \mathrm{m} / \mathrm{z}$ calc'd for $\mathrm{C}_{12} \mathrm{H}_{25} \mathrm{NOS}_{2} \mathrm{SiNa}^{+}$: 314.1039; found: 314.1040 .
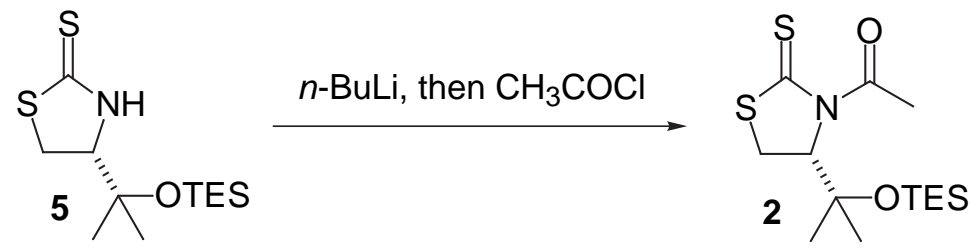

Thiazolidinethione 5 (5.10 g, $17.44 \mathrm{mmol})$ was dissolved in THF (100 $\mathrm{mL})$ and cooled to $-78{ }^{\circ} \mathrm{C}$. $n$-BuLi (13.08 mL, $20.93 \mathrm{mmol}, 1.6 \mathrm{M}$ in hexane) was then added dropwise, and the reaction was stirred at $-78{ }^{\circ} \mathrm{C}$ for $30 \mathrm{~min}$. Acetyl chloride $(1.49 \mathrm{~mL}, 20.93 \mathrm{mmol})$ was then added dropwise, and the reaction was stirred at $-78^{\circ} \mathrm{C}$ for 1 hour, and then warmed to room temperature over a period of $40 \mathrm{~min}$ and stirred for additional $30 \mathrm{~min}$. The reaction was quenched by the addition of aqueous $\mathrm{NH}_{4} \mathrm{Cl}$ (saturated solution, $20 \mathrm{~mL}$ ) and the aqueous layer was further extracted with $\mathrm{CH}_{2} \mathrm{Cl}_{2}(2 \times 50 \mathrm{~mL})$. The combined organic layers were dried over anhydrous $\mathrm{MgSO}_{4}$, filtered and then concentrated at reduced pressure to a yellow oil. Purification by flash chromatography (10:1 hexanes:EtOAc) gave thiazolidinethione $\mathbf{2}$ as a clear, yellow oil (5.24 g, $90 \%)$.

${ }^{1} \mathrm{H}$ NMR $\left(500 \mathrm{MHz}, \mathrm{CDCl}_{3}\right): \delta 5.26(\mathrm{X}$ of $\mathrm{ABX}, 1 \mathrm{H}, \mathrm{J}=1.01,8.34 \mathrm{~Hz}), 3.45(\mathrm{~A}$ of $\mathrm{ABX}$, $1 \mathrm{H}, \mathrm{J}=8.34,11.45 \mathrm{~Hz}), 3.35(\mathrm{~B}$ of $\mathrm{ABX}, 1 \mathrm{H}, \mathrm{J}=1.01,11.45 \mathrm{~Hz}), 2.74(\mathrm{~s}, 3 \mathrm{H}), 1.28(\mathrm{~s}, 3 \mathrm{H}), 1.26,(\mathrm{~s}$, $3 \mathrm{H}), 0.92(\mathrm{t}, 9 \mathrm{H}, \mathrm{J}=7.80 \mathrm{~Hz}), 0.58(\mathrm{q}, 6 \mathrm{H}, \mathrm{J}=7.80 \mathrm{~Hz}) .{ }^{13} \mathrm{C}$ NMR $\left(400 \mathrm{MHz}, \mathrm{CDCl}_{3}\right): \delta 205.8$, 170.6, 76.9, 72.5, 30.2, 28.2, 27.0, 26.6, 7.3, 6.8. IR $\left(\mathrm{cm}^{-1}\right): 1693,1009$. [ $\left.\alpha\right]_{\mathrm{D}}=-344.18(\mathrm{c} 1.00$, EtOH). Anal. Calcd for $\mathrm{C}_{14} \mathrm{H}_{27} \mathrm{NO}_{2} \mathrm{~S}_{2} \mathrm{Si}: \mathrm{C}, 50.41 ; \mathrm{H}, 8.16 ; \mathrm{N}, 4.20$, Found: $\mathrm{C}, 50.69 ; \mathrm{H}, 7.78 ; \mathrm{N}$, 
4.60 .

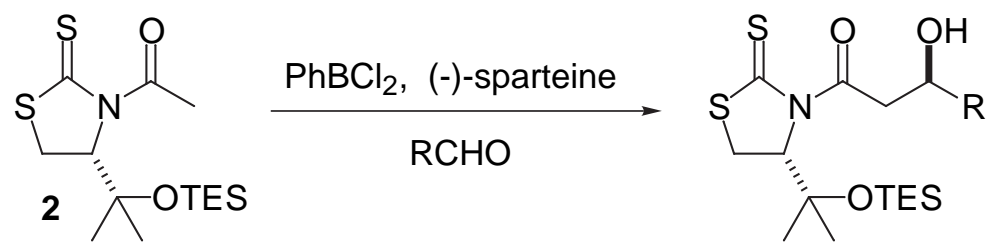

Typical aldol procedure:

To a $10 \mathrm{~mL}$ round-bottom flask was added $N$-acetyl thiazolidinethione $\mathbf{2}(120 \mathrm{mg}, 0.360$ mmol, $1.30 \mathrm{eq})$ and $\mathrm{CH}_{2} \mathrm{Cl}_{2}(1.5 \mathrm{~mL})$. The flask was cooled to $0{ }^{\circ} \mathrm{C}$ and $\mathrm{PhBCl}_{2}(47.8 \mu \mathrm{L}, 0.360$ mmol, $1.30 \mathrm{eq}$ ) was then added dropwise to give an orange colored solution. After stirring for 10 min, (-)-sparteine $(0.165 \mathrm{~mL}, 0.720 \mathrm{mmol}, 2.6$ eq.) was added dropwise. Upon addition of the sparteine, the reaction turned yellow. The ice bath was removed, and the reaction was allowed to warm to room temperature and stirr for $30 \mathrm{~min}$. The reaction was then cooled to $-78{ }^{\circ} \mathrm{C}$ and the aldehyde $(0.277 \mathrm{mmol}, 1.0 \mathrm{eq}$.) was added dropwise. The reaction was then stirred for $6 \mathrm{~h}$ at -78 ${ }^{\circ} \mathrm{C}$, and then slowly warmed to $0{ }^{\circ} \mathrm{C}$ over a period of about $3 \mathrm{~h}$ by allowing the dry ice to evaporate. Once the temperature reached $0{ }^{\circ} \mathrm{C}$, the reaction was placed in an ice bath and stirred for $30 \mathrm{~min}$. The reaction was then quenched by the addition of hexanes $(3 \mathrm{~mL})$ and $\mathrm{H}_{2} \mathrm{O}_{2}(30 \%, 1.5 \mathrm{~mL})$, and stirred rapidly for $10 \mathrm{~min}$ at $0{ }^{\circ} \mathrm{C}$. (Note that rapid stirring is required at this step. If the rate of stirring is too slow, incomplete oxidation of the borane occurs, and boron species are observed in the product.) The solution was diluted with hexanes and $\mathrm{CH}_{2} \mathrm{Cl}_{2}(4: 1,80 \mathrm{~mL})$, and the layers were separated. The organic layer was further washed with distilled water and brine, dried over anhydrous $\mathrm{MgSO}_{4}$, filtered, and concentrated at reduced pressure to an orange oil. Analysis of this material by ${ }^{1} \mathrm{H}$ NMR provided the d.r. values given in Table 1 . The products were purified by flash chromatography using neutral silica gel (1:1 hexanes : $\mathrm{CH}_{2} \mathrm{Cl}_{2}$ to 1:4 hexanes : $\mathrm{CH}_{2} \mathrm{Cl}_{2}$ depending on substrate, see general information for details regarding the use of neutral silica gel) to provide the aldol adducts as clear yellow oils. Note that the thiazolidinethione is sensitive to hydrolysis, and that this work-up has been optimized to minimize hydrolysis, and should be followed carefully in order to obtain high yields and reproducible results.

Table 1, entry 1:

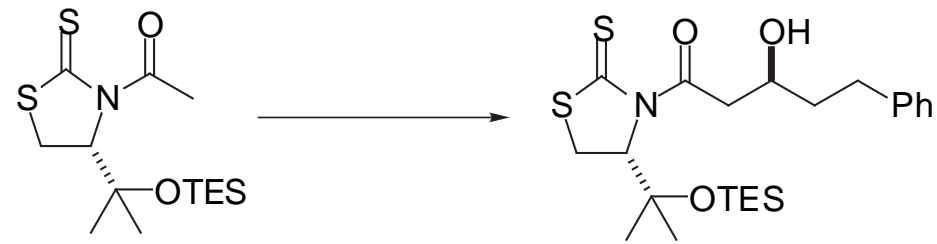

${ }^{1} \mathrm{H}$ NMR $\left(500 \mathrm{MHz}, \mathrm{C}_{6} \mathrm{D}_{6}\right): \delta 7.30-7.16(\mathrm{~m}, 5 \mathrm{H}), 5.28(\mathrm{X}$ of $\mathrm{ABX}, 1 \mathrm{H}, \mathrm{J}=1.37,8.00 \mathrm{~Hz})$, $4.02(\mathrm{~m}, 1 \mathrm{H}), 3.49$ (A of ABX, 1H, J=9.70, $17.39 \mathrm{~Hz}), 3.45$ (A of ABX, 1H, J=8.00, 11.44 Hz), $3.40(\mathrm{~B}$ of $\mathrm{ABX}, 1 \mathrm{H}, \mathrm{J}=1.37,11.44 \mathrm{~Hz}), 3.36(\mathrm{~d}, \mathrm{OH}, \mathrm{J}=4.03 \mathrm{~Hz}), 3.27(\mathrm{~B}$ of $\mathrm{ABX}, 1 \mathrm{H}, \mathrm{J}=2.66$, $17.39 \mathrm{~Hz}), 2.82(\mathrm{~m}, 1 \mathrm{H}), 2.70(\mathrm{~m}, 1 \mathrm{H}), 1.89(\mathrm{~m}, 1 \mathrm{H}), 1.76(\mathrm{~m}, 1 \mathrm{H}), 1.29(\mathrm{~s}, 3 \mathrm{H}), 1.26,(\mathrm{~s}, 3 \mathrm{H}), 0.93$ $(\mathrm{t}, 9 \mathrm{H}, \mathrm{J}=7.90 \mathrm{~Hz}), 0.58(\mathrm{q}, 6 \mathrm{H}, \mathrm{J}=7.90 \mathrm{~Hz}) .{ }^{13} \mathrm{C}$ NMR $\left(400 \mathrm{MHz}, \mathrm{C}_{6} \mathrm{D}_{6}\right): \delta 205.9,173.3,142.0$, 128.7, 128.6, 126.1, 72.4, 67.8, 45.0, 38.4, 31.9, 30.4, 28.3, 26.6, 7.3, 6.8. IR ( $\left.\mathrm{cm}^{-1}\right): 3437,1646$, 1615, 1033. $[\alpha]_{D}=-269.866\left(\mathrm{c} 0.94\right.$, EtOH). Anal. Calcd for $\mathrm{C}_{23} \mathrm{H}_{37} \mathrm{NO}_{3} \mathrm{~S}{ }_{2} \mathrm{Si}: \mathrm{C}, 59.06 ; \mathrm{H}, 7.97$; N, 2.99, Found: C, 59.31; H, 7.73; N, 3.39.

Table 1, entry 2: 


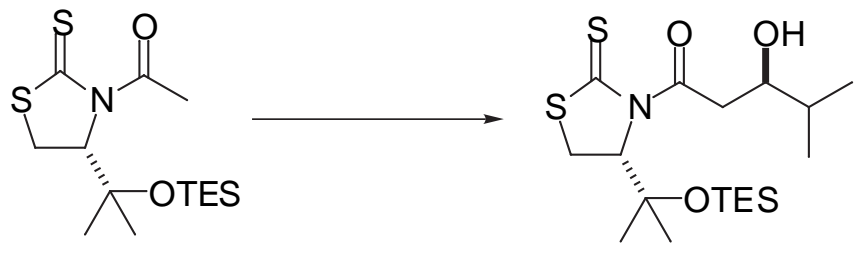

${ }^{1} \mathrm{H}$ NMR $\left(500 \mathrm{MHz}, \mathrm{CDCl}_{3}\right): \delta 5.31(\mathrm{X}$ of $\mathrm{ABX}, 1 \mathrm{H}, \mathrm{J}=1.30,8.10 \mathrm{~Hz}), 3.78(\mathrm{~m}, 1 \mathrm{H}), 3.53$ (A of ABX, 1H, 8.50, 16.85 Hz), 3.45 (A of ABX, 1H, J=8.10, 11.53 Hz), 3.39 (B of ABX, 1H, $\mathrm{J}=1.30,11.53 \mathrm{~Hz}), 3.23(\mathrm{~d}, \mathrm{OH}, \mathrm{J}=5.04 \mathrm{~Hz}), 3.22(\mathrm{~B}$ of $\mathrm{ABX}, 1 \mathrm{H}, \mathrm{J}=1.30,16.85 \mathrm{~Hz}), 1.74(\mathrm{~m}, 1 \mathrm{H})$, $1.29(\mathrm{~s}, 3 \mathrm{H}), 1.27,(\mathrm{~s}, 3 \mathrm{H}), 0.94(\mathrm{~d}, 3 \mathrm{H}, \mathrm{J}=6.77 \mathrm{~Hz}), 0.92(\mathrm{~d}, 3 \mathrm{H}, \mathrm{J}=6.77 \mathrm{~Hz}), 0.91(\mathrm{t}, 9 \mathrm{H}, \mathrm{J}=7.78$ $\mathrm{Hz}), 0.59(\mathrm{q}, 6 \mathrm{H}, \mathrm{J}=7.78 \mathrm{~Hz}) .{ }^{13} \mathrm{C} \mathrm{NMR}\left(400 \mathrm{MHz}, \mathrm{C}_{6} \mathrm{D}_{6}\right): \delta 206.2,173.7,76.9,73.6,72.5,41.9$, 33.7, 30.3, 28.3, 26.5, 18.7, 18.1, 7.3, 6.8. IR $\left(\mathrm{cm}^{-1}\right): 3440,1685,1153$. [ $\left.\alpha\right]_{\mathrm{D}}=-271.436(\mathrm{c} 0.84$, $\mathrm{EtOH}) . \mathrm{HRMS} \mathrm{m} / \mathrm{z}$ calc'd for $\mathrm{C}_{18} \mathrm{H}_{35} \mathrm{NO}_{3} \mathrm{~S}_{2} \mathrm{SiNa}^{+}$: 428.1719 ; found: 428.1715 .

Table 1, entry 3:

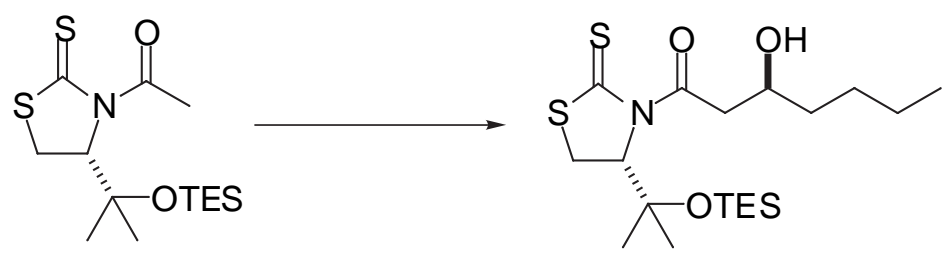

${ }^{1} \mathrm{H}$ NMR $\left(500 \mathrm{MHz}, \mathrm{C}_{6} \mathrm{D}_{6}\right): \delta 5.30(\mathrm{X}$ of ABX, 1H, J=1.28, 8.10 Hz), $3.99(\mathrm{~m}, 1 \mathrm{H}), 3.46$ (A of ABX, 1H, J=8.15, 17.31 Hz), 3.45 (A of ABX, 1H, J=8.10, 11.54 Hz), 3.39 (B of ABX, 1H, $\mathrm{J}=1.28,11.54 \mathrm{~Hz}), 3.27$ (B of ABX, 1H, J=1.28, $17.31 \mathrm{~Hz}), 3.24($ br s, OH), $1.55(\mathrm{~m}, 2 \mathrm{H}), 1.45$ (m, 2H), $1.29(\mathrm{~s}, 3 \mathrm{H}), 1.28(\mathrm{~m}, 2 \mathrm{H}), 1.26(\mathrm{~s}, 3 \mathrm{H}), 0.92(\mathrm{t}, 9 \mathrm{H}, \mathrm{J}=7.78 \mathrm{~Hz}), 0.86(\mathrm{t}, 3 \mathrm{H}, \mathrm{J}=7.33 \mathrm{~Hz}), 0.59$ $(\mathrm{q}, 6 \mathrm{H}, \mathrm{J}=7.78 \mathrm{~Hz}) .{ }^{13} \mathrm{C} \mathrm{NMR}\left(400 \mathrm{MHz}, \mathrm{C}_{6} \mathrm{D}_{6}\right) ; \delta 205.9,173.4,76.9,72.4,68.7,44.9,36.5,30.3$, 28.3, 27.9, 26.6, 22.8, 14.2, 7.3, 6.8. IR $\left(\mathrm{cm}^{-1}\right): 3417,1639,1153$. [ $\left.\alpha\right]_{\mathrm{D}}=-233.188(\mathrm{c} 1.28$, EtOH). HRMS m/z calc'd for $\mathrm{C}_{19} \mathrm{H}_{37} \mathrm{NO}_{3} \mathrm{~S}_{2} \mathrm{SiNa}^{+}$: 442.1876; found: 442.1864 .

Table 1, entry 4:

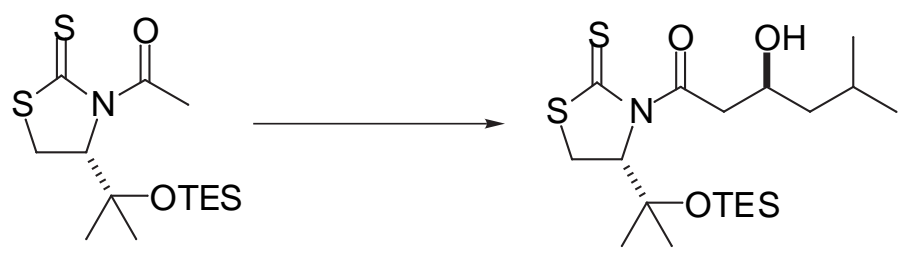

${ }^{1} \mathrm{H}$ NMR $\left(500 \mathrm{MHz}, \mathrm{C}_{6} \mathrm{D}_{6}\right): \delta 5.30(\mathrm{X}$ of $\mathrm{ABX}, 1 \mathrm{H}, \mathrm{J}=1.00,8.13 \mathrm{~Hz}), 4.08(\mathrm{~m}, 1 \mathrm{H}), 3.46$ (A of ABX, 1H, J=9.66, 17.14 Hz), 3.44 (A of ABX, 1H, J=8.13, 11.65 Hz), 3.39 (B of ABX, 1H, $\mathrm{J}=1.00,11.65 \mathrm{~Hz}), 3.22(\mathrm{~B}$ of $\mathrm{ABX}, 1 \mathrm{H}, \mathrm{J}=2.53,17.14 \mathrm{~Hz}), 3.20(\mathrm{br} \mathrm{s}, \mathrm{OH}), 1.79(\mathrm{~m}, 1 \mathrm{H}), 1.52(\mathrm{~m}$, $1 \mathrm{H}), 1.28(\mathrm{~s}, 3 \mathrm{H}), 1.25,(\mathrm{~s}, 3 \mathrm{H}), 1.21(\mathrm{~m}, 1 \mathrm{H}), 0.92(\mathrm{t}, 9 \mathrm{H}, \mathrm{J}=8.00 \mathrm{~Hz}), 0.89$ (d, 3H, J=1.31Hz), 0.88 $(\mathrm{d}, 3 \mathrm{H}, \mathrm{J}=1.31 \mathrm{~Hz}), 0.58(\mathrm{q}, 6 \mathrm{H}, \mathrm{J}=8.00 \mathrm{~Hz}) .{ }^{13} \mathrm{C}$ NMR $\left(400 \mathrm{MHz}, \mathrm{C}_{6} \mathrm{D}_{6}\right): \delta 205.9,173.4,72.4$, $66.8,45.9,45.4,30.3,28.3,26.5,24.6,23.5,22.3,7.3,6.8$. IR $\left(\mathrm{cm}^{-1}\right): 3448,1681,1149 . \quad[\alpha]_{\mathrm{D}}=$ -258.536 (c 1.35, EtOH). HRMS m/z calc'd for $\mathrm{C}_{19} \mathrm{H}_{37} \mathrm{NO}_{3} \mathrm{~S}_{2} \mathrm{SiNa}^{+}$: 442.1876; found: 442.1895.

Table 1, entry 5: 


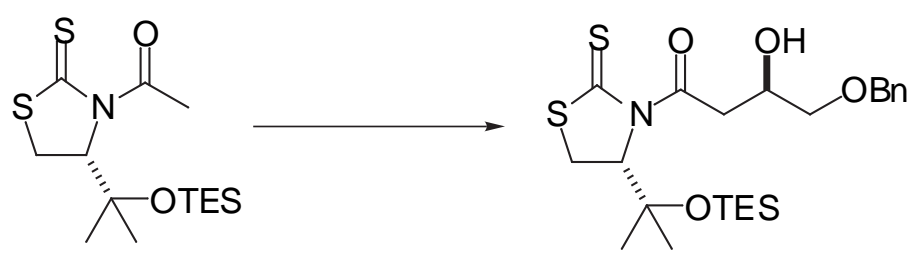

${ }^{1} \mathrm{H}$ NMR (500 MHz, $\left.\mathrm{CDCl}_{3}\right): \delta 7.34-7.25(\mathrm{~m}, 5 \mathrm{H}), 5.27(\mathrm{X}$ of ABX, $1 \mathrm{H}, \mathrm{J}=1.50,8.00 \mathrm{~Hz})$, $4.55(\mathrm{dd}, 2 \mathrm{H}, \mathrm{J}=12.09,14.29), 4.25(\mathrm{~m}, 1 \mathrm{H}), 3.59$ (A of ABX, 1H, J=8.00, $17.49 \mathrm{~Hz}), 3.50$ (d, 2H, $\mathrm{J}=5.22 \mathrm{~Hz}$ ), $3.44(\mathrm{~A}$ of $\mathrm{ABX}, 1 \mathrm{H}, \mathrm{J}=8.00,11.54 \mathrm{~Hz}), 3.38(\mathrm{~B}$ of $\mathrm{ABX}, 1 \mathrm{H}, \mathrm{J}=1.50,11.54 \mathrm{~Hz}), 3.37$ $(\mathrm{B}$ of $\mathrm{ABX}, 1 \mathrm{H}, \mathrm{J}=1.50,17.49 \mathrm{~Hz}), 3.23(\mathrm{~d}, \mathrm{OH}, \mathrm{J}=4.58 \mathrm{~Hz}), 1.28(\mathrm{~s}, 3 \mathrm{H}), 1.26,(\mathrm{~s}, 3 \mathrm{H}), 0.93(\mathrm{t}, 9 \mathrm{H}$, $\mathrm{J}=7.78 \mathrm{~Hz}$ ), 0.59 (q, 6H, J=7.78 Hz). ${ }^{13} \mathrm{C} \mathrm{NMR}\left(400 \mathrm{MHz}, \mathrm{CDCl}_{3}\right): \delta 205.7,172.6,138.2,128.7$, 127.9, 73.5, 73.2, 72.5, 67.8, 42.0, 30.3, 28.3, 26.5, 7.3, 6.8. IR $\left(\mathrm{cm}^{-1}\right): 3431,1685,1448,1150$. $[\alpha]_{D}=-143.374\left(\mathrm{c} 1.03\right.$, EtOH). Anal. Calcd for $\mathrm{C}_{23} \mathrm{H}_{37} \mathrm{NO}_{4} \mathrm{~S}_{2} \mathrm{Si}: \mathrm{C}, 57.10 ; \mathrm{H}, 7.71 ; \mathrm{N}, 2.90$, Found: C, 57.29; H, 7.63; N, 3.22.

Table 1, entry 6:

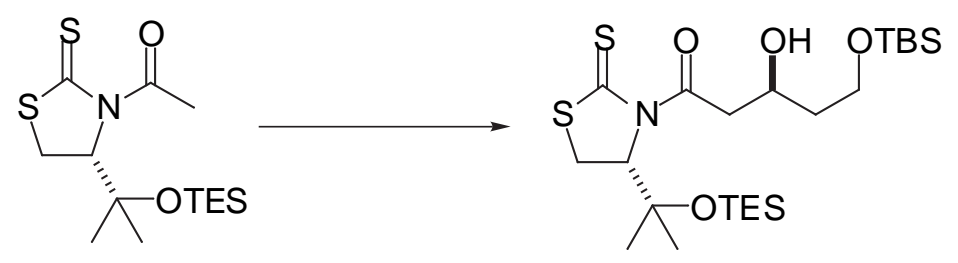

${ }^{1} \mathrm{H}$ NMR $\left(500 \mathrm{MHz}, \mathrm{CDCl}_{3}\right): \delta 5.29(\mathrm{X}$ of ABX, 1H, J=1.19, $8.00 \mathrm{~Hz}), 4.24(\mathrm{~m}, 1 \mathrm{H}), 3.80$ $(\mathrm{m}, 2 \mathrm{H}), 3.58(\mathrm{~d}, \mathrm{OH}, \mathrm{J}=3.66 \mathrm{~Hz}), 3.49$ (A of ABX, 1H, J=8.40, 17.40 Hz), 3.45 (A of ABX, 1H, $\mathrm{J}=8.00,11.53 \mathrm{~Hz}$ ), 3.38 (B of ABX, 1H, J=1.19, 11.53Hz), 3.33 (B of ABX, 1H, J=1.50, 17.40 $\mathrm{Hz}), 1.71(\mathrm{~m}, 2 \mathrm{H}), 1.28(\mathrm{~s}, 3 \mathrm{H}), 1.26,(\mathrm{~s}, 3 \mathrm{H}), 0.92(\mathrm{t}, 9 \mathrm{H}, \mathrm{J}=7.87 \mathrm{~Hz}), 0.86(\mathrm{~s}, 9 \mathrm{H}), 0.59(\mathrm{q}, 6 \mathrm{H}$, $\mathrm{J}=7.87 \mathrm{~Hz}), 0.04(\mathrm{~s}, 6 \mathrm{H}) .{ }^{13} \mathrm{C} \mathrm{NMR}\left(400 \mathrm{MHz}, \mathrm{CDCl}_{3}\right): \delta 205.7,172.7,72.5,67.1,61.1,45.3$, $38.8,30.3,28.3,26.5,26.1,18.5,7.3,6.8,-5.2$. IR $\left(\mathrm{cm}^{-1}\right): 3433,1673,1153 .[\alpha]_{\mathrm{D}}=-126.828(\mathrm{c}$ 1.30, EtOH). Anal. Calcd for $\mathrm{C}_{22} \mathrm{H}_{47} \mathrm{NO}_{4} \mathrm{~S}_{2} \mathrm{Si}_{2}: \mathrm{C}, 52.93 ; \mathrm{H}, 9.08 ; \mathrm{N}, 2.68$, Found: C, 53.30; H, $8.62 ; \mathrm{N}, 3.02$.

Table 1, entry 7:

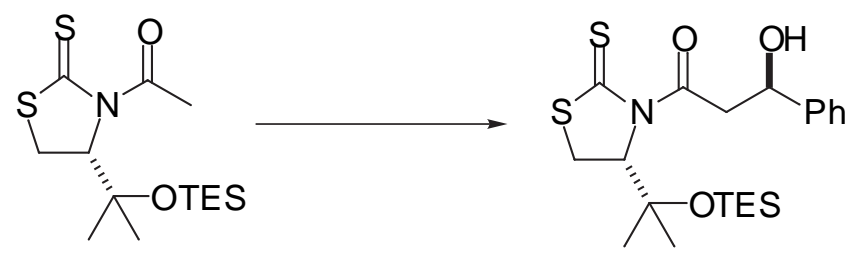

${ }^{1} \mathrm{H}$ NMR (500 MHz, $\left.\mathrm{CDCl}_{3}\right): \delta$ 7.40-7.25 (m, 5H), 5.30 (X of ABX, 1H, J=0.88, 8.00), $5.12(\mathrm{~m}, 1 \mathrm{H}), 3.85(\mathrm{~A}$ of $\mathrm{ABX}, 1 \mathrm{H}, \mathrm{J}=9.89,17.36 \mathrm{~Hz}), 3.70(\mathrm{~d}, \mathrm{OH}, \mathrm{J}=4.39 \mathrm{~Hz}), 3.51$ (B of ABX, $1 \mathrm{H}, \mathrm{J}=2.85,17.36 \mathrm{~Hz}), 3.45(\mathrm{~A}$ of $\mathrm{ABX}, 1 \mathrm{H}, \mathrm{J}=8.00,11.43 \mathrm{~Hz}), 3.39$ (B of ABX, 1H, J=0.88, $11.43 \mathrm{~Hz}), 1.30(\mathrm{~s}, 3 \mathrm{H}), 1.27,(\mathrm{~s}, 3 \mathrm{H}), 0.93(\mathrm{t}, 9 \mathrm{H}, \mathrm{J}=8.00 \mathrm{~Hz}), 0.59$ (q, 6H, J=8.00 Hz). ${ }^{13} \mathrm{C}$ NMR $\left(400 \mathrm{MHz}, \mathrm{C}_{6} \mathrm{D}_{6}\right): \delta 205.8,172.6,142.6,128.7,127.9,126.1,72.5,71.0,46.4,30.4,28.2,26.6,7.3$, 6.8. IR $\left(\mathrm{cm}^{-1}\right): 3437,1642,1448,1141 .[\alpha]_{\mathrm{D}}=-257.812$ (c 1.81, EtOH). HRMS m/z calc'd for $\mathrm{C}_{21} \mathrm{H}_{33} \mathrm{NO}_{3} \mathrm{~S}_{2} \mathrm{SiNa}^{+}$: 462.1563; found: 462.1538 .

Table 1, entry 8: 
Page S-7

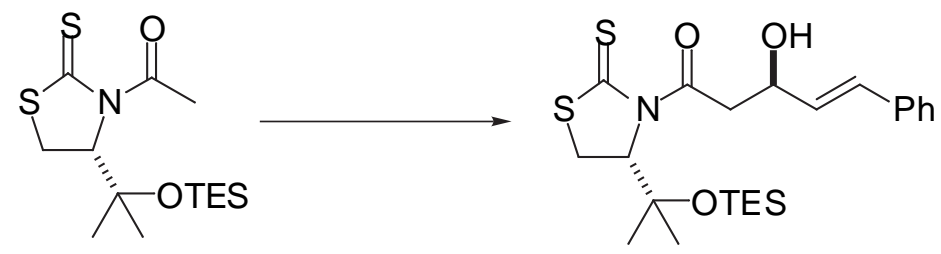

${ }^{1} \mathrm{H} \mathrm{NMR}\left(500 \mathrm{MHz}, \mathrm{CDCl}_{3}\right): \delta 7.38-7.21(\mathrm{~m}, 5 \mathrm{H}), 6.65(\mathrm{dd}, 1 \mathrm{H}, \mathrm{J}=0.88,16.00 \mathrm{~Hz}), 6.25$ (dd, 1H, J=5.94, 16.00), 5.30 (X of ABX, 1H, J=1.10, 8.13 Hz), $4.74(\mathrm{~m}, 1 \mathrm{H}), 3.72(\mathrm{~A}$ of ABX, $1 \mathrm{H}$, $\mathrm{J}=9.01,17.14 \mathrm{~Hz}$ ), 3.46 ( $\mathrm{A}$ of $\mathrm{ABX}, 1 \mathrm{H}, \mathrm{J}=8.13,11.43 \mathrm{~Hz}), 3.42$ (B of $\mathrm{ABX}, 1 \mathrm{H}, \mathrm{J}=3.29,17.14$ ), 3.43 (br, s, OH), 3.41 (B of ABX, 1H, J=1.10, $11.43 \mathrm{~Hz}$ ), 1.30 (s, 3H), 1.27, (s, 3H), 0.93 (t, 9H, $\mathrm{J}=8.00 \mathrm{~Hz}), 0.58(\mathrm{q}, 6 \mathrm{H}, \mathrm{J}=8.00 \mathrm{~Hz}) .{ }^{13} \mathrm{C} \mathrm{NMR}\left(400 \mathrm{MHz}, \mathrm{C}_{6} \mathrm{D}_{6}\right): \delta 205.9,172.5,136.7,131.0$, 130.3, 128.8, 128.0, 126.8, 72.5, 69.5, 44.8, 30.4, 28.3, 26.6, 7.3, 6.8. IR (cm $\left.{ }^{-1}\right): 3421,1638,1033$. $[\alpha]_{\mathrm{D}}=-296.158(\mathrm{c} 0.59, \mathrm{EtOH})$. HRMS m/z calc'd for $\mathrm{C}_{23} \mathrm{H}_{35} \mathrm{NO}_{3} \mathrm{~S}_{2} \mathrm{SiNa}^{2}: 488.1719$; found: 488.1714 . 